\title{
CT Imaging in the Diagnosis of Lung Injury of Organophosphorus Poisoning and Analysis of Its Correlation with Procalcitonin and C-Reactive Protein Levels
}

\author{
Wenwen Sun $\mathbb{D}^{1,2}$, Dongliang Wang $\mathbb{D}^{2},{ }^{2}$ and Xianliang Yan $\mathbb{D}^{1}$ \\ ${ }^{1}$ Department of Emergency Medicine, The Affiliated Hospital of Xuzhou Medical University, Xuzhou 221002, Jiangsu, China \\ ${ }^{2}$ Emergency Medical Center, Suqian First People's Hospital Affiliated to Nanjing Medical University, Suqian 223800, \\ Jiangsu, China
}

Correspondence should be addressed to Xianliang Yan; 11231538@stu.wxic.edu.cn

Received 30 August 2021; Revised 12 October 2021; Accepted 15 October 2021; Published 1 November 2021

Academic Editor: Gustavo Ramirez

Copyright (C) 2021 Wenwen Sun et al. This is an open access article distributed under the Creative Commons Attribution License, which permits unrestricted use, distribution, and reproduction in any medium, provided the original work is properly cited.

This study focused on the application value of CT images of patients with organophosphorus poisoning lung injury. Specifically, 50 patients diagnosed with organophosphorus pesticide poisoning lung injury in the hospital were selected as research subjects. They all had chest CT examinations. Patients were classified according to different degrees of poisoning, and different groups were compared for the levels of C-reactive protein (CRP) and procalcitonin (PCT) in the serum. The results showed that, of the 50 patients, 25 patients had mild organophosphorus poisoning, accounting for $50 \%, 14$ patients had moderate poisoning, accounting for $28 \%$, and 11 patients suffered from severe organophosphorus poisoning. It was found that, in AOPP patients, cascade effect was exerted by stimulating the secretion of inflammatory mediators such as CRP, causing systemic inflammatory response syndrome, and multiorgan failure appeared in the early stage of the disease. After the histogram equalization treatment, the image was clearer than that processed by the other three methods. In terms of CT image smoothing, the effect of the Gaussian filtering was better than the mean filtering effect, and the effect of the median filtering was better than that of the Gaussian filtering. Therefore, the structure of the lungs became clearer, and the lung texture was also clearly displayed, and CT images after histogram equalization have an important value in the diagnosis of lung injury caused by organophosphorus poisoning.

\section{Introduction}

Organophosphorus poisoning is common in China. In all poisoning cases that occur each year, acute organophosphorus poisoning (AOPP) accounts for $1 / 5-1 / 2$, and the fatality rate of patients after poisoning is as high as $40 \%$ $[1,2]$. Organophosphorus is mainly used in agriculture and has insecticidal effects. Because people do not use a mask to cover their mouth, nose, and skin when using organophosphorus pesticides, it is easy for the pesticides to enter the human body and eventually poison people [3]. The mechanism of AOPP is mainly related to acetylcholinesterase (AChE). Organophosphorus pesticides can reduce the activity of AChE [4]. Studies have shown that the secretion of inflammatory mediators such as $\mathrm{C}$-reactive protein (CRP) in AOPP patients is increased to exert the cascade effect, which leads to the occurrence of systemic inflammatory response syndrome (SIRS) [5-7]. CRP is a protein related to acute inflammations and has high sensitivity and specificity. Higher content of hypersensitive CRP will lead to a heavier inflammatory response and a higher probability of complications $[8,9]$. Detecting the concentration of CRP in the patient's serum can evaluate the condition, make an early diagnosis, and provide guidance for the treatment $[10,11]$. Under normal conditions, the concentration of procalcitonin (PCT) in the body is low. When the body has an emergency such as an infection, the level of PCT will be significantly increased, especially in bacterial-induced sepsis [12]. It is believed that serum PCT is one of the markers of organophosphorus poisoning patients with early bacterial 
TABLE 1: Basic information of organophosphorus poisoning patients $(n=50)$.

\begin{tabular}{|c|c|c|c|}
\hline Index & & Number (case) & Proportion (\%) \\
\hline \multirow{2}{*}{ Gender } & Male & 37 & 74 \\
\hline & Female & 13 & 26 \\
\hline \multirow{2}{*}{ Age } & $>50$ years old & 33 & 66 \\
\hline & $\leq 50$ years old & 17 & 34 \\
\hline \multirow{2}{*}{ Whether to aspirate by mistake } & Yes & 5 & 10 \\
\hline & No & 45 & 90 \\
\hline \multirow{2}{*}{ Ventilator use } & Yes & 14 & 28 \\
\hline & No & 36 & 72 \\
\hline \multirow{2}{*}{ Time to stay in the intensive care unit } & $>3$ days & 8 & 16 \\
\hline & $\leq 3$ days & 42 & 84 \\
\hline \multirow{2}{*}{ Cholinesterase activity } & $>15 \%$ & 34 & 68 \\
\hline & $\leq 15 \%$ & 16 & 32 \\
\hline \multirow{2}{*}{ High-sensitivity CRP } & $>6.5 \mathrm{mg} / \mathrm{L}$ & 48 & 96 \\
\hline & $\leq 6.5 \mathrm{mg} / \mathrm{L}$ & 2 & 4 \\
\hline
\end{tabular}

infections to induce multiorgan inflammations, and it has sensitivity and specificity [13]. Clinical studies have shown that patients with organophosphorus poisoning combined with respiratory failure can easily lead to a decline in the body's immune level, and long-term hospitalization can easily lead to lung infections, and the mortality rate will also be increased, thus threatening the life and health of patients $[14,15]$. For patients with organophosphorus poisoning combined with respiratory failure, antibiotics are routinely used for treatment, and the effect is significant [16].

In the above, CT imaging is of great significance for the diagnosis of organophosphorus poisoning lung injury. In this study, organophosphorus poisoning patients of different degrees were compared for the serum CRP and PCT levels, expected to provide a reference for the early diagnosis of organophosphorus poisoning lung injury.

\section{Materials and Methods}

2.1. Basic Information of the Subjects. This study was a retrospective study. From January 2019 to February 2021, 50 patients in the hospital who were diagnosed as having suspected inhaled organophosphorus poisoning were selected as research subjects. All of the 50 patients underwent the CT examination and were examined for the plasma levels, the content of CRP and PCT. This study did not require patients to sign an informed consent form, and this study was approved by the ethics committee of the hospital.

The inclusion criteria were as follows: (a) meet the diagnostic criteria of organophosphorus pesticide poisoning; (b) complete clinical data of patients; (c) patients and their family members cooperate with various examinations and treatments.

The exclusion criteria were as follows: (a) the patient was previously diagnosed with other lung diseases, such as chronic obstructive pulmonary disease (COPD), pneumonia, lung cancer, and tuberculosis; (b) those with unclear CT images; (c) those with other underlying diseases; (d) pregnant and breastfeeding patients. Of the 50 patients, 40 patients were randomly selected for feature extraction of imaging omics, and the remaining 10 patients were used for model verification.
2.2. Detection of CRP and PCT. Fifty patients were randomly divided into the AOPP group and control group. In the AOPP group, there were 14 males and 11 females. The age ranged from 20 to 63 years, with an average of $35.3 \pm 6.2$ years. In the control group, there were 16 males and 9 females. The average age was $35.6 \pm 6.0$ years from 21 to 64 years. The time of poisoning was $0.3-3.5 \mathrm{~h}$, with an average of $1.1 \pm 0.3 \mathrm{~h}$. Severity: mild in 25 cases, moderate in 17 cases, and severe in 8 cases. There was no significant difference in gender and age between the two groups $(1.1 \pm 0.05)$, with comparability. According to different AOPP groups and control groups, $2 \mathrm{ml}$ of venous blood was collected within $2 \mathrm{~h}$ after admission, and serum hypersensitive CRP concentration was determined by immunoturbidimetry. Serum PCT concentration and serum PCT and CRP levels were determined by semiquantitative solid-phase immunochromatography. Among them, serum PCT was quantitatively detected by the double-antibody sandwich method, and serum CRP was quantitatively detected by immunoturbidimetry. $12 \mu \mathrm{L}$ of serum was extracted and added into the dilution tube, which was detected by QuikRead go.

2.3. Statistical Analysis. In this study, SPSS 22.0 software was used for data processing. Measurement data were expressed as mean \pm standard deviation, and count data were expressed as a percentage. $P<0.05$ indicated that the difference was statistically significant.

\section{Results}

3.1. Basic Information of the Subjects. In this study, 50 patients who were diagnosed as having suspected organophosphorus poisoning in the hospital from June 2019 to April 2021 were selected as research subjects. Of the 50 patients, 37 were males and 13 were females, with an average age of 56.8 years. The basic information of the research subjects of this study is shown in Table 1.

\subsection{Classification of Severity of Organophosphorus Poisoning.} Of the 50 patients, according to the degree of organophosphorus pesticide poisoning, they were classified as mild, moderate, and severe. The degree of poisoning was classified 


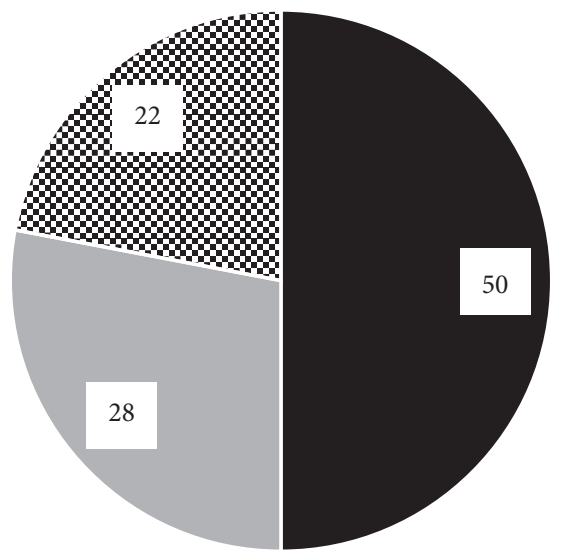

- A: mildly poisoned by organophosphorus

- B: moderately poisoned by organophosphate

\% C: severely poisoned by organophosphate

Figure 1: Distribution of the severity of organophosphate in 50 patients.

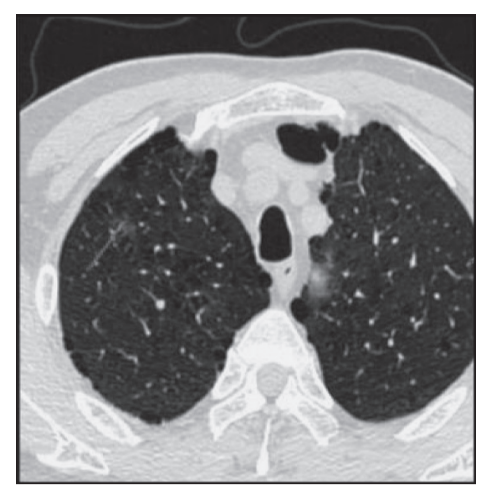

(a)

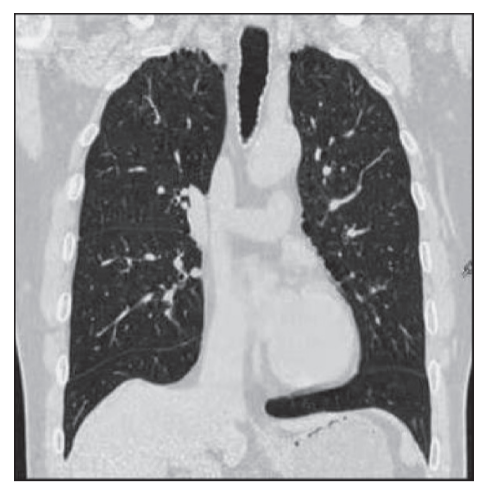

(d)

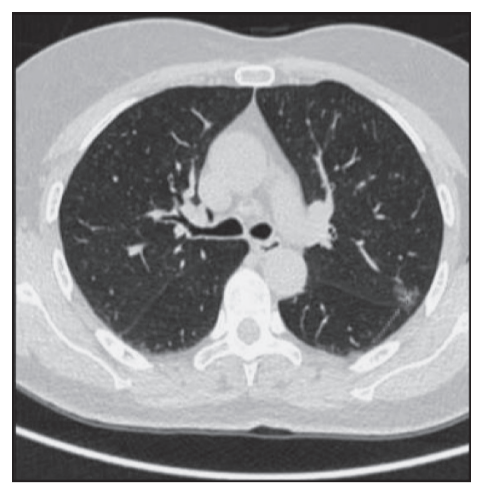

(b)

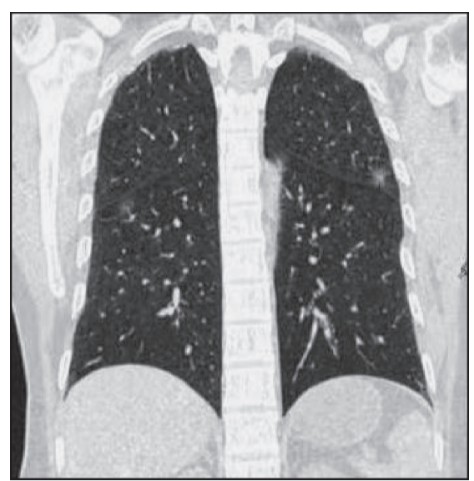

(e)

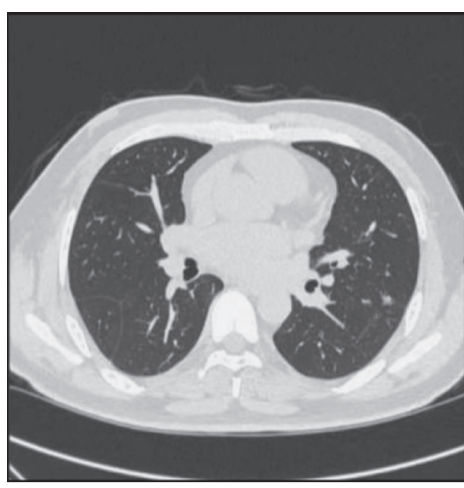

(c)

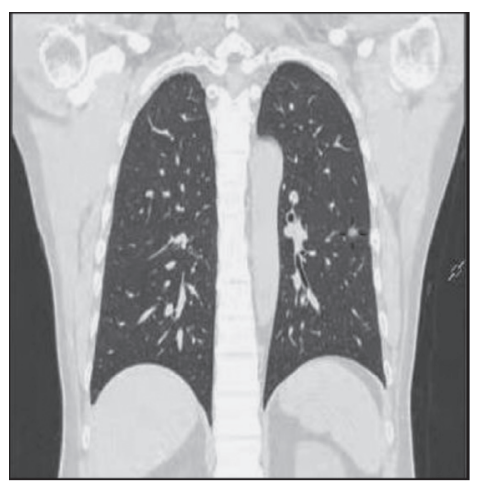

(f)

Figure 2: Continued. 


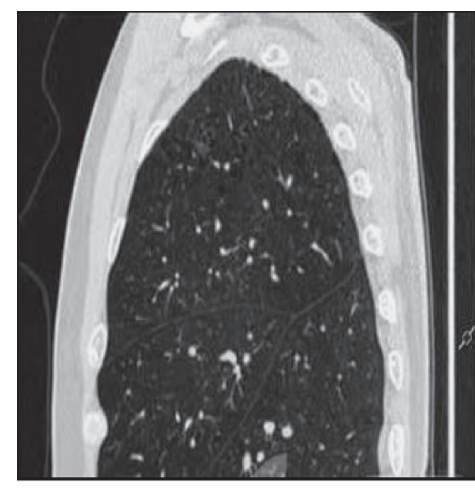

(g)

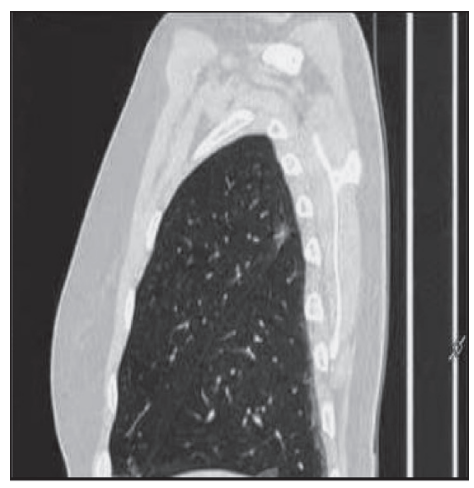

(h)

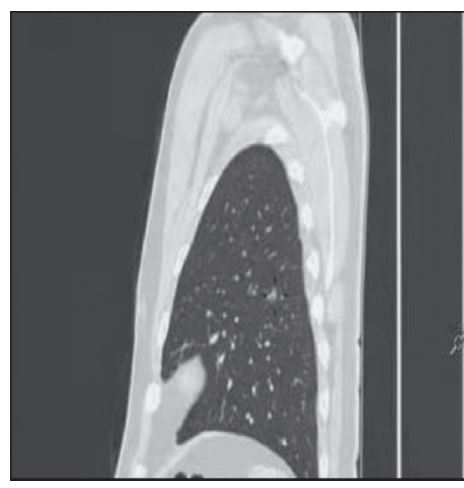

(i)

FIgURE 2: Original CT plain scan image: $(\mathrm{a}-\mathrm{c})$ a transverse lung CT image; $(\mathrm{d}-\mathrm{f})$ a coronal lung CT image; (g-i) a coronal lung CT image. The red arrows in this image indicate where the lung lesions are located.

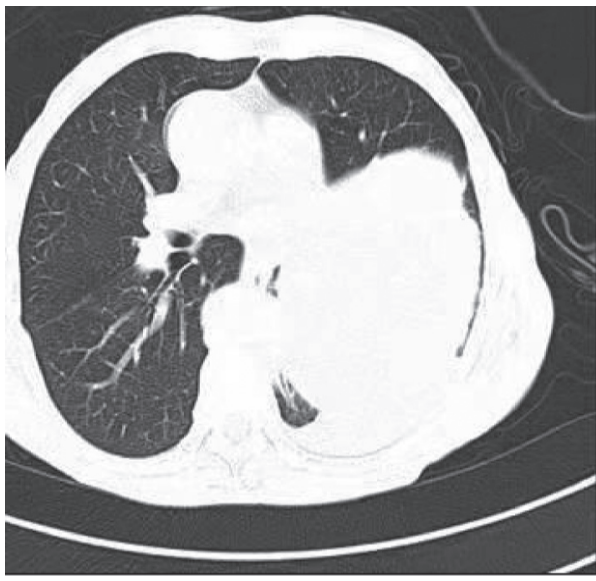

(a)

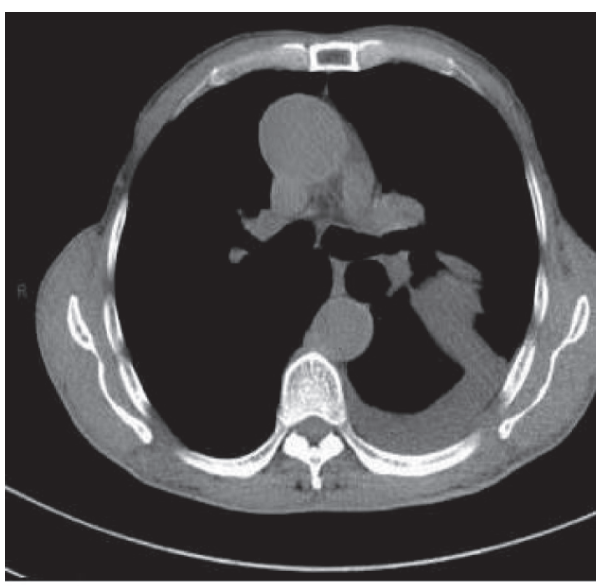

(c)

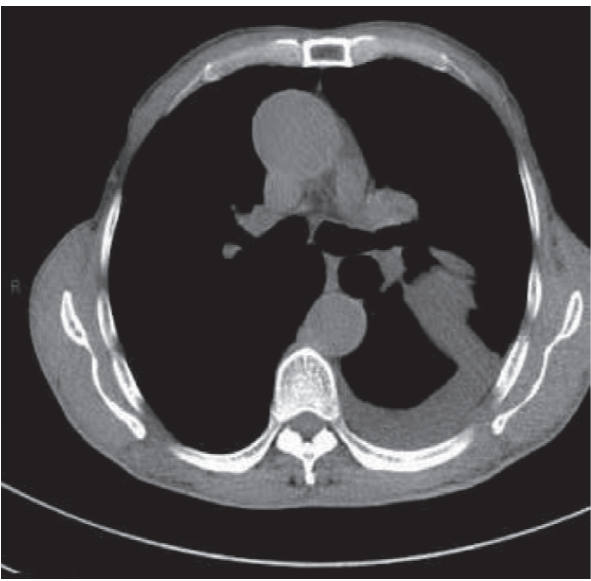

(b)

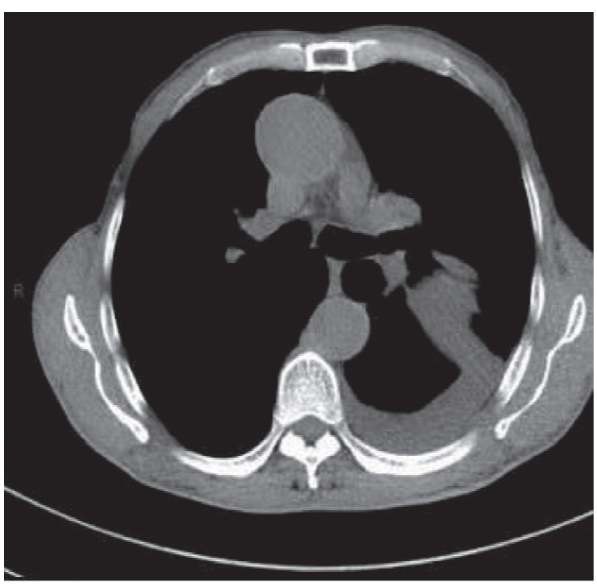

(d)

FIGURE 3: CT image after CT smoothing: (a) the original image; (b) the image after the mean filter processing; (c) the image after Gaussian filtering; (d) the image after median filtering.

according to the degree of decrease in blood cholinesterase activity. Mild: a decrease of 50\%-70\%, moderate: a decrease of $30 \%-50 \%$, and severe: decrease of less than $30 \%$. Of the 50 patients, 25 were mildly intoxicated, accounting for 50\%, 14 were moderately intoxicated, accounting for $28 \%$, and there were 11 severe patients, as shown in Figure 1. 


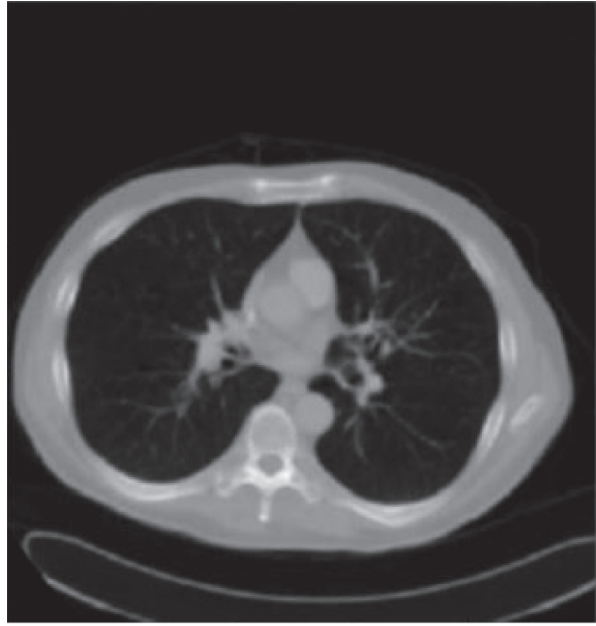

(a)

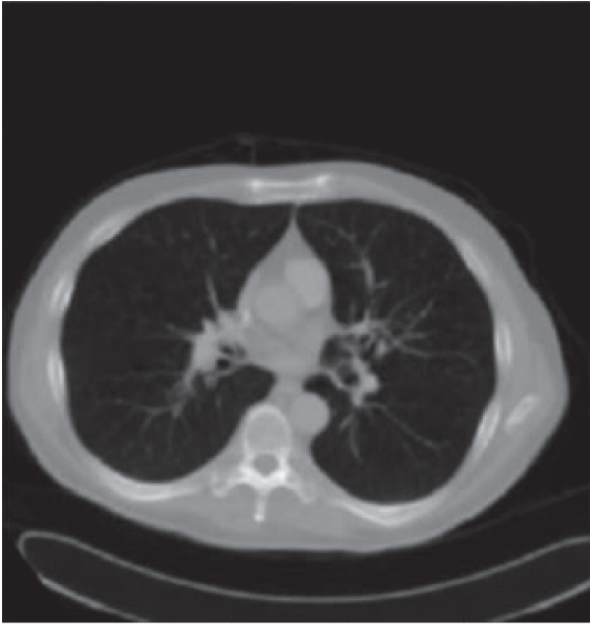

(b)

FIGURE 4: CT image after histogram equalization processing: (a) the original CT image; (b) the CT image after equalization.

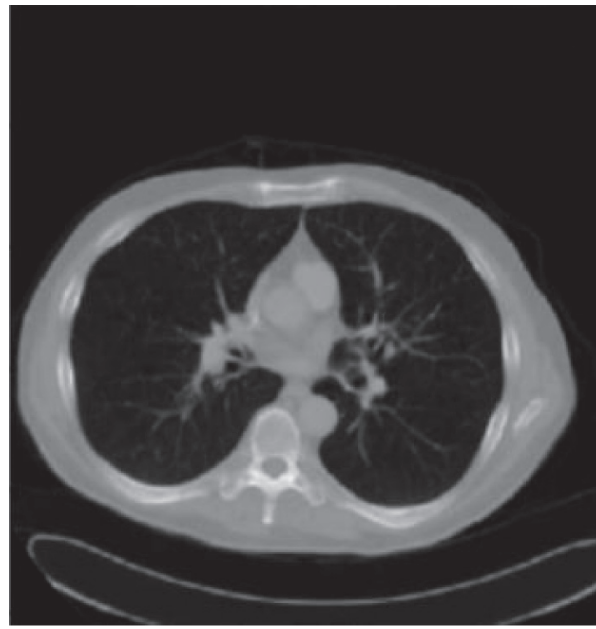

(a)

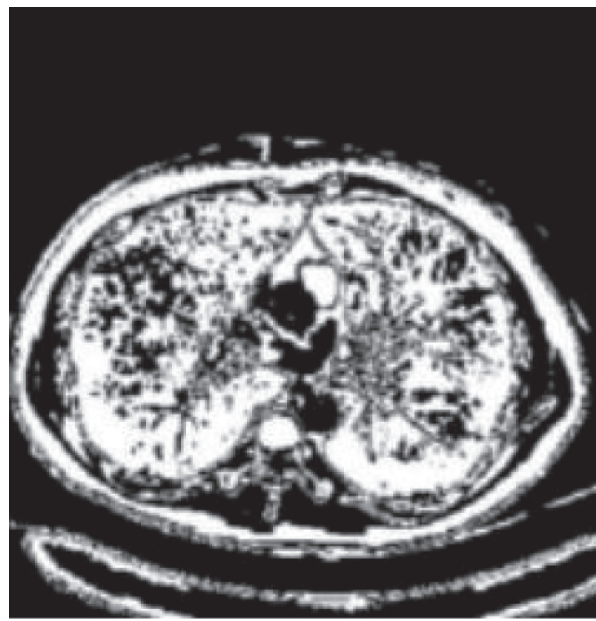

(c)

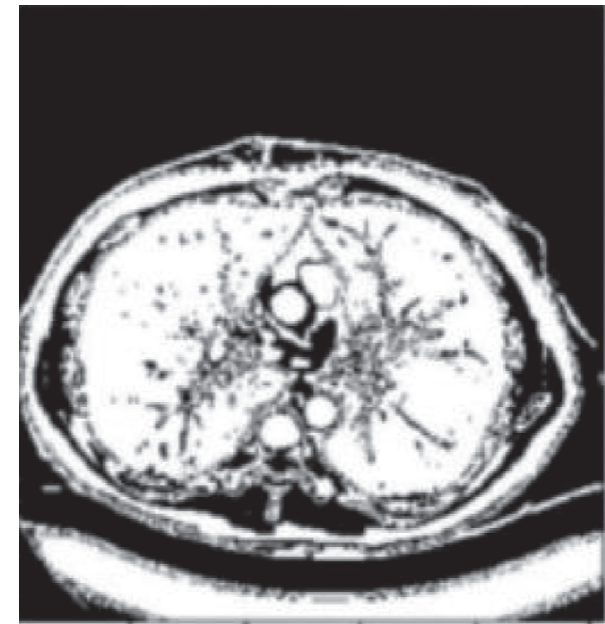

(b)

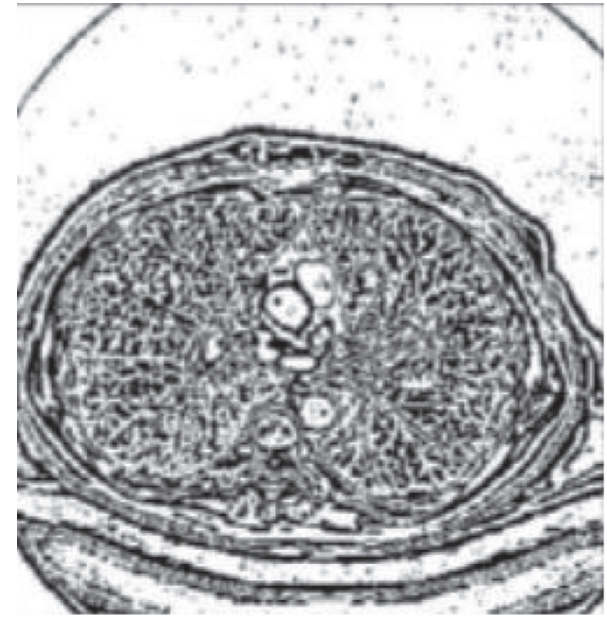

(d)

FIGURE 5: CT image after binarization processing: (a) the initial CT image; (b) the CT image that underwent Otsu threshold binarization processing; (c) the one undergoing fixed binarization processing; (d) the CT image undergoing adaptive binarization processing. 


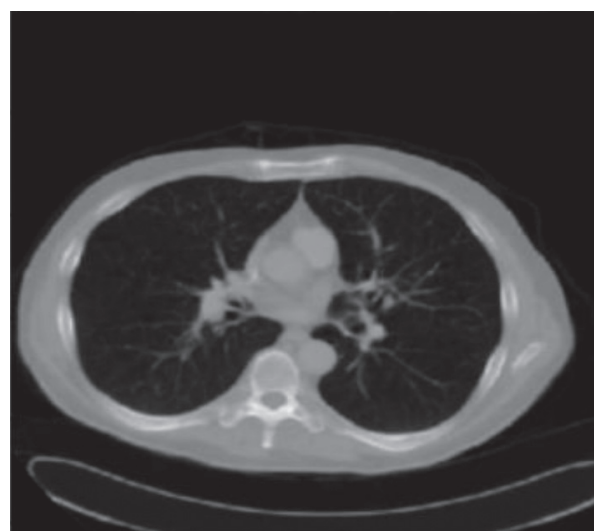

(a)

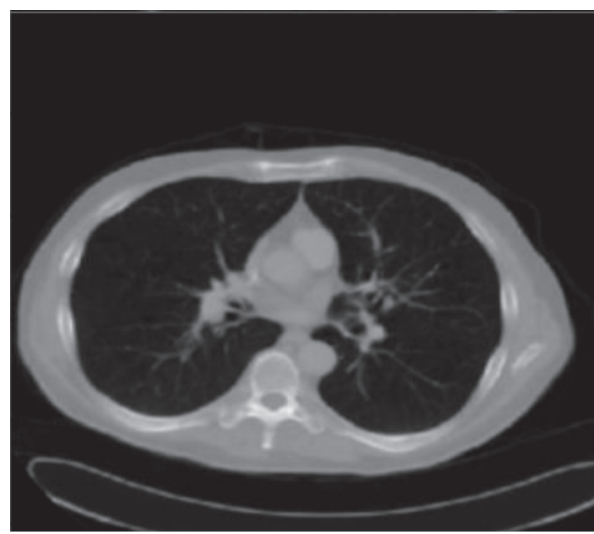

(c)

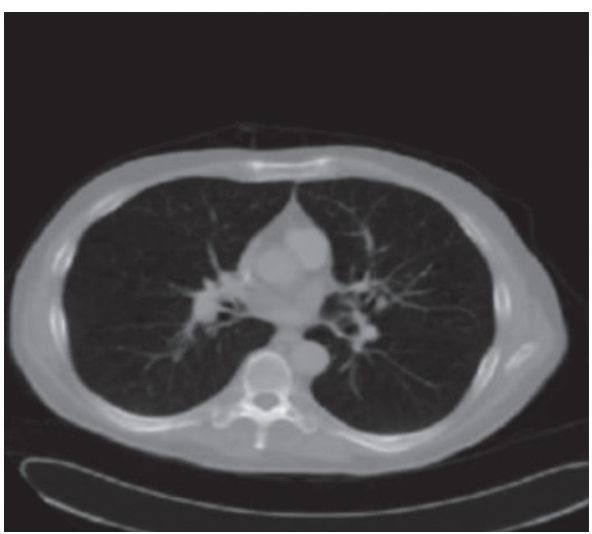

(b)

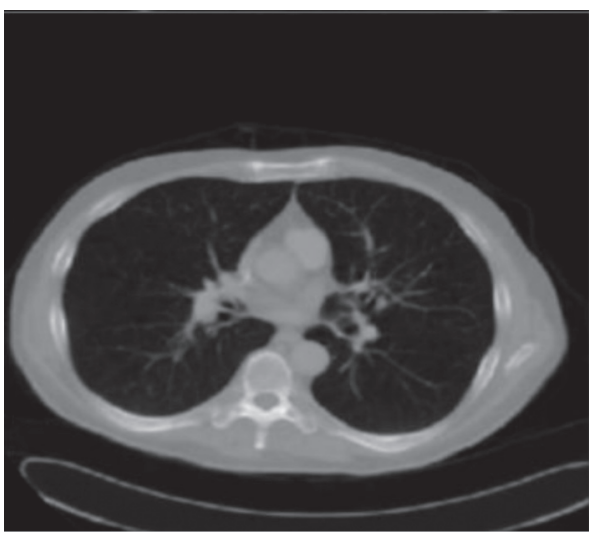

(d)

FIgURE 6: CT image processed by mathematical morphology: (a) the CT image processed by the corrosion operation; (b) the CT image processed by the expansion operation; (c) the CT image processed by the opening operation; (d) the CT image processed by the closing operation.

3.3. CT Images of Patients with Organophosphorus Poisoning Lung Injury. Due to different times of organophosphorus poisoning, the chest CT images between individuals were different. The main manifestations were as follows: (a) ground-glass density shadow; (b) lung exudation and consolidation shadow; (c) pulmonary interstitial fibrosis; (d) bronchiectasis; (e) mediastinal and subcutaneous emphysema; and (f) pleural and pericardial effusion. The CT scan results are shown in Figure 2.

\subsection{Image Data Preprocessing}

3.4.1. CT Image Smoothing. In terms of CT image smoothing, the original CT image was processed in three ways: mean filtering, Gaussian filtering, and median filtering. It was found that the effect of Gaussian filtering was better than that of mean filtering, and the effect of median filtering was better than that of Gaussian filtering. For this reason, median filtering was used for smoothing, as shown in Figure 3.

3.4.2. CT Image Equalization Processing. After the image underwent histogram equalization, the structure of the lungs was clearer, and the lung texture was also clearly displayed, as shown in Figure 4.

3.4.3. Binarization of CT Images. Three methods of Otsu, fixed threshold, and adaptive threshold were used to process the original CT images. The processed results are shown in Figure 5 .

3.4.4. Mathematical Morphology Processing. After the histogram equalization processing, there was still some noise in the lung area, which needed to be processed by the Shuchun morphological method. After processing, it was noted that the image was clearer than that processed by the other three methods, as shown in Figure 6.

3.5. PCT and CRP in Patients with Organophosphorus Poisoning Lung Injury. According to the severity of organophosphorus poisoning, patients were divided into the mild group, moderate group, and severe group, and the number of patients was 25,14 , and 11 , respectively. Figure 7 shows the hypersensitivity CRP and PCT of the three groups of patients. 

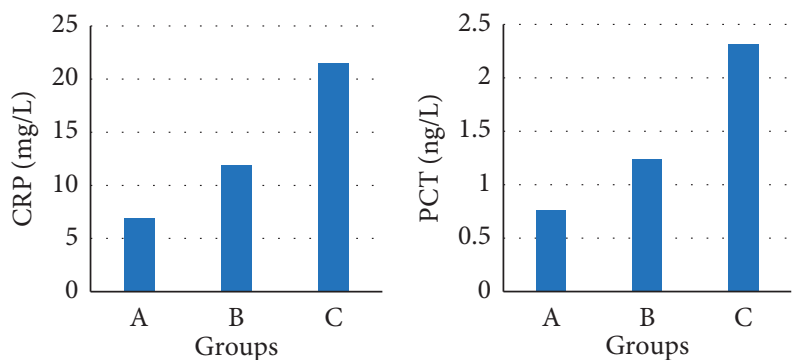

FIgURE 7: Comparison of hypersensitivity CRP and PCT levels. Note: $\mathrm{A}$ is the mild group; $\mathrm{B}$ is the moderate group; $\mathrm{C}$ is the severe group.

\section{Discussion}

The occurrence of AOPP is related to the inactivation of AChE. As a result, a large amount of acetylcholine accumulates at the cholinergic receptor site, resulting in continuous high-intensity excitement of the cholinergic nerves, which affects the central and peripheral nervous systems [17]. In this study, patients with organophosphorus poisoning were divided into mild, moderate, and severe groups according to the activity of cholinesterase, and the differences between their CRP and PCT levels were compared. The results showed that, of the 50 patients, 25 patients had mild organophosphorus poisoning, accounting for 50\%, 14 patients were moderately poisoned, accounting for $28 \%$, and there were 11 severe patients. It has been found that, in AOPP patients, cascade effect is exerted by stimulating the secretion of inflammatory mediators such as CRP, causing systemic inflammatory response syndrome, and multiorgan failure appears in the early stage of the disease [18]. Adenosine triphosphate and reactive oxygen species produced by mitochondria play a key role in disease progression, and these two substances can also promote cell apoptosis. Mitochondrial damage can lead to cardiovascular, liver, inflammation, and neurodegenerative diseases. The leakage of electrons in the mitochondrial respiratory chain generates ROS, which causes oxidative damage [19].

When organophosphorus enters the body, it causes multiple-organ damage, and nerves are in an emergency state of hyperactivity. The hypersecretion of the pancreas leads to a sharp increase in blood amylase activity, thus increasing the blood glucose level. Leukocytes and CRP are often used as indicators of inflammation and acute injury in clinical practice. In patients with organophosphorus poisoning, vascular endothelial cells are damaged, resulting in the adhesion of monocyte-macrophages and T lymphocytes, and the monocyte-lymphocyte system of the body is activated, resulting in the explosive release of leukocyte inflammatory factors, leading to systemic inflammatory response syndrome. As an acute protein that rises rapidly when the body is injured or infected, CRP plays a very important role in the acute reaction and immune response of organophosphorus poisoning patients. After the absorption of organophosphorus, the activity of AChE is also sharply decreased, and the decomposition of acetylcholine is weakened, leading to abnormal accumulation of acetylcholine in the body, affecting the cholinergic mechanism of the body and producing a series of poisoning symptoms. The results of this study suggested that, as the organophosphorus poisoning worsens, the levels of CRP and PCT in patients showed a gradual upward trend.

\section{Conclusion}

CT images of patients with organophosphorus severe lung injury were optimized in this study, and then patients with organophosphorus pesticide poisoning of different degrees were compared for the levels of CRP and PCT in the serum. Next, the relationship between CT image characteristics and the content of CRP and PCT in the patient's serum was analyzed. It elevated the diagnostic accuracy of patients with organophosphorus poisoning lung injury. Additionally, the CT image processed by it was clearer and better reflected the details in the CT image, so it has high theoretical and practical significance. However, some limitations in the study should be noted. The sample size is small, which will reduce the power of the study. In the follow-up, expanded sample size is necessary to strengthen the findings of the study.

\section{Data Availability}

The data used to support the findings of this study are available from the corresponding author upon request.

\section{Conflicts of Interest}

The authors declare that there are no conflicts of interest.

\section{Acknowledgments}

This work was supported by the Xuzhou National Clinical Key Specialty Cultivation Project (2018ZK004), Jiangsu Provincial Commission of Health and Family Planning (LGY2019085), Excellent Young and Middle-Age Talents Project of the Affiliated Hospital of Xuzhou Medical University (2019128009), and National Key Point Research and Invention Program (2020YFC1512704).

\section{References}

[1] E. J. Hulse, J. D. Haslam, S. R. Emmett, and T. Woolley, "Organophosphorus nerve agent poisoning: managing the poisoned patient," British Journal of Anaesthesia, vol. 123, no. 4, pp. 457-463, 2019.

[2] S. R. Bajracharya, P. N. Prasad, and R. Ghimire, "Management of organophosphorus poisoning," Journal of Nepal Health Research Council, vol. 14, no. 34, pp. 131-138, 2016.

[3] P. Jacquet, L. Poirier, D. Daudé, and E. Chabrière, "Intoxication aux organophosphorés: vers des traitements enzymatiques," Annales Pharmaceutiques Françaises, vol. 77, no. 5, pp. 349-362, 2019, French.

[4] M. Eddleston, "Novel clinical toxicology and pharmacology of organophosphorus insecticide self-poisoning," Annual Review of Pharmacology and Toxicology, vol. 59, no. 1, pp. 341-360, 2019. 
[5] M. Eddleston and F. R. Chowdhury, "Organophosphorus poisoning: the wet opioid toxidrome," The Lancet, vol. 397, no. 10270, pp. 175-177, 2021.

[6] N. R. Sproston and J. J. Ashworth, "Role of C-reactive protein at sites of inflammation and infection," Frontiers in Immunology, vol. 9, p. 754, 2018.

[7] E. M. Dyer, T. Waterfield, and H. Baynes, "How to use C-reactive protein," Archives of disease in childhood - Education \& practice edition, vol. 104, no. 3, pp. 150-153, 2019.

[8] M. Moutachakkir, A. Lamrani Hanchi, A. Baraou, A. Boukhira, and S. Chellak, "Immunoanalytical characteristics of C-reactive protein and high sensitivity C-reactive protein," Annales de Biologie Clinique, vol. 75, no. 2, pp. 225-229, 2017.

[9] L. Badimon, E. Peña, G. Arderiu et al., "C-reactive protein in atherothrombosis and angiogenesis," Frontiers in Immunology, vol. 9, p. 430, 2018.

[10] C. Bray, L. N. Bell, H. Liang et al., "Erythrocyte sedimentation rate and C-reactive protein measurements and their relevance in clinical medicine," Wisconsin Medical Journal: Official Publication of the State Medical Society of Wisconsin, vol. 115, no. 6, pp. 317-321, 2016.

[11] L. Hu, Q. Shi, M. Shi, R. Liu, and C. Wang, "Diagnostic value of PCT and CRP for detecting serious bacterial infections in patients with fever of unknown origin: a systematic review and meta-analysis," Applied Immunohistochemistry \& Molecular Morphology, vol. 25, no. 8, pp. e61-e69, 2017.

[12] J. H. Tang, D. P. Gao, and P. F. Zou, "Comparison of serum PCT and CRP levels in patients infected by different pathogenic microorganisms: a systematic review and meta-analysis," Brazilian journal of medical and biological research $=$ Revista brasileira de pesquisas medicas e biologicas, vol. 51, no. 7, Article ID e6783, 2018.

[13] B. E. Belsher, E. Beech, D. Evatt et al., "Present-centered therapy (PCT) for post-traumatic stress disorder (PTSD) in adults," Cochrane Database of Systematic Reviews, vol. 2019, no. 11, Article ID CD012898, 2019.

[14] R. Sager, A. Kutz, B. Mueller, and P. Schuetz, "Procalcitoninguided diagnosis and antibiotic stewardship revisited," $B M C$ Medicine, vol. 15, no. 1, p. 15, 2017.

[15] E. Aloisio, A. Dolci, and M. Panteghini, "Procalcitonin: between evidence and critical issues," Clinica Chimica Acta, vol. 496, pp. 7-12, 2019.

[16] A. K. Singal, "Porphyria cutanea tarda: recent update," Molecular Genetics and Metabolism, vol. 128, no. 3, pp. 271-281, 2019.

[17] M. Melough, X. Sun, and O. Chun, "The role of AOPP in agerelated bone loss and the potential benefits of berry anthocyanins," Nutrients, vol. 9, no. 7, p. 789, 2017.

[18] M. Kawami, T. Shimonakamura, R. Yumoto, and M. Takano, "Transport of AOPP-albumin into human alveolar epithelial A549 cell," Journal of Pharmacy \& Pharmaceutical Sciences, vol. 21, no. 1, pp. 247-255, 2018.

[19] L. Lin, G. J. Zhao, and L. L. Qin, "Association between advanced oxidation protein products (AOPP) and vascular calcification in uremic patients," European Review for Medical and Pharmacological Sciences, vol. 21, no. 18, pp. 4147-4152, 2017. 